\title{
Sabemos cómo impacta la selección y contratación de un talento humano para la organización?
}

\author{
¿Do we know how the selection and hiring of a human talent \\ impacts the organization?
}

\begin{abstract}
RESUMEN
Actualmente las organizaciones están pasando por cambios disruptivos, como la globalización de negocios, desarrollo tecnológico y desarrollo del talento humano que conllevan a generar mayor competitividad entre las organizaciones. Es así, que las empresas ante el fuerte impacto del cambio y el intenso movimiento de las organizaciones en busca de la calidad y productividad, se están enfocando en mejorar sus procesos de selección de personal por competencias para captar y seleccionar talentos humanos que agreguen valor, innovación y rentabilidad a la empresa. Por tanto, el objetivo del artículo, es relevar la importancia de los procesos de selección de competencias, y su impacto para la organización, la contratación de personal competente, revestido talento humano para el logro de ventajas competitivas únicas y sostenibles frente a la competencia. Es por eso, que, en los tiempos actuales, el modelo de selección por competencias tiene mayor relevancia en las competencias y habilidades que las empresas exigen a los profesionales, para que sean capaces de afrontar con éxito los retos del futuro, y así adaptarse a cambios internos y externos. Por el tipo de investigación, el presente estudio empleada es de tipo descriptivo, porque se basa en la descripción y/o narración de las características de un objeto observado; y de tipo Correlación, porque mide el grado de relación que existe entre dos o más variables.
\end{abstract}

Palabras Clave: Selección; Competencias; Talento Humano.

\begin{abstract}
Organizations are currently undergoing disruptive changes, such as business globalization, technological development and development of human talent that lead to greater competitiveness among organizations. Thus, companies, faced with the strong impact of change and the intense movement of organizations in search of quality and productivity, are focusing on improving their personnel selection processes based on competencies to attract and select human talents that add value, innovation and profitability to the company. Therefore, the objective of the article is to highlight
\end{abstract}

Presentado: 30/11/2020 - Aceptado: 30/04/2021 - Publicado: 23/12/2021

(C) Los autores. Este artículo es publicado por la revista Gestión en el Tercer Milenio de la Facultad de Ciencias Administrativas Universidad Nacional Mayor de San Marcos. Este es un artículo de acceso abierto, distribuido bajo los términos de la licencia Creative Commons Atribución 4.0 Internacional (CC BY 4.0) [https://creativecommons.org/licenses/by/4.0/deed.es] que permite el uso, distribución y reproducción en cualquier medio, siempre que la obra original sea debidamente citada de su fuente original. 
the importance of the processes of selection of competencies, and their impact for the organization, the hiring of competent personnel, equipped with human talent for the achievement of unique and sustainable competitive advantages over the competition. That is why, in current times, the competency selection model has greater relevance in the competencies and skills that companies require from professionals, so that they are able to successfully face the challenges of the future, and thus adapt to internal and external changes. Due to the type of research, the present study used is descriptive, because it is based on the description and / or narration of the characteristics of an observed object; and of type Correlation, because it measures the degree of relationship that exists between two or more variables.

Keywords: Selection; Competences; Human Talent.

\section{INTRODUCCIÓN}

Dado el contexto actual, la administración de las personas es una de las áreas que más ha sufrido cambios y transformaciones. En los años recientes, el mundo ha cambiado radicalmente y con ellos las personas y las organizaciones donde no existen fronteras y el mundo se ha digitalizado generando mayor competitividad entre los países y las organizaciones. En este contexto, las empresas progresivamente se han ido acomodando a estos cambios, para lo cual dentro de sus ventajas competitivas, consideran al recurso humano como elemento clave dentro de este proceso, que ha conllevado a buscar que disponer de personal que sea el más idóneo y competitivo para ocupar una posición específica dentro de la organización, teniendo en cuenta sus capacidades y competencias actuales y potencialidades, capaz de adaptarse a cualquier cambio que se pueda dar dentro de la organización o en su entorno externo.

Esto implica que ahora las empresas no solo deben de pensar en contratar personal para que ocupen un puesto, sino a seleccionar a la persona idónea que genere valor agregado a la organización; por lo que las organizaciones deben disponer de procesos modernos y adecuados de selección de personal, que van desde los procesos de convocatoria, captación, búsqueda de talentos, hasta los procesos internos de evaluación, selección y contratación, que permita tomar la mejor decisión, es decir: "seleccionar a los mejores o más competentes o ambas características que sería los más idóneo". No olvidar, que uno de los elementos claves dentro de estos procesos, es definir apropiadamente el perfil del puesto relacionadas con las características y competencias requeridas, y el potencial del mismo, sugiero: de ello depende todo el proceso de búsqueda, captación, selección y contratación de personal.

\section{OBJETIVO DEL ARTÍCULO}

El presente artículo tiene como propósito relevar la importancia e impacto estratégico que una organización puede obtener, si cuenta con recurso humano idóneo y competitivo para el logro de sus objetivos y metas organizacionales, considerando el mundo globalizado tan cambiante y dinámico en su evolución, que conlleva a permanentes disrupciones dentro y fuera de las organizaciones y solo las organizaciones que estén preparadas para adaptarse a estos cambios serán las que finalmente sobrevivirán.

Bajo este propósito, es imprescindible que las organizaciones dentro de sus objetivos estratégicos, prioricen el área de recursos humanos, considerándose como la columna vertebral de cualquier organización, porque ahí se llevan a cabo los procesos de selección y contratación de personal, basados en el modelo de gestión por competencias, que incluya los procesos de reclutamiento, selección y contratación por competencias para la contratación de personal idóneo y competitivo que permita generar valor y hacer más competitiva a la organización frente a su capacidad, dentro del sector dentro del cual se desarrolla.

Por tanto, el disponer de un modelo de selección por competencias que se ajuste al modelo y tipo de la organización, en relación a sus políticas, estructura y el alineamiento con los procesos con sus objetivos organizacionales, 
permiten asegurar el desarrollo de procesos de calidad orientados a la convocatoria, selección y contratación de personal idóneo y competitivo (talento humanos) capaces de generar impactos basados en un nivel de competencia superior, generar y adaptarse a estos cambios en la organización y ventajas competitivas dentro de nuestro sector.

\section{ARGUMENTACIÓN}

Todos somos conscientes que los procesos de reclutamiento, selección y contratación de personal son de vital importancia para cualquier empresa, para captar y contratar al personal idóneo que agregue valor a la empresa frente a sus competidores. Por tanto, podemos señalar sin temor a equivocarnos, que la competitividad de las empresas depende en gran medida, del personal competente con talento humano que la conforman, que garantizan la eficiencia, innovación y la mayor productividad en el desarrollo de sus actividades.

Los recursos humanos constituyen el talón de Aquiles de las políticas de modernización, esto significa cambiar la cultura de la organización y por tanto, incidir sobre valores y actitudes de las personas que trabajan en la empresa y como es conocido, hoy más que nunca, las organizaciones deberán ser generadoras de aprendizaje, para formar y desarrollar capacidades en los recursos humanos que les permitirá capitalizar el conocimiento, lograr sus objetivos y metas con un recurso humano dotado de ciertas habilidades y un perfil idóneo que la hará competitiva y la ayudará a contrarrestar las amenazas y detectar las oportunidades. (Ruiz et al., 2017, p. 10)

Entonces, queda claro que las empresas deben priorizar dentro de sus objetivos estratégicos, los objetivos y estrategias de selección de personal, que conllevan a la empresa disponer dentro de sus Oficinas de Recursos Humanos de políticas, procesos, y procedimientos claros y alineados, personal competente y capacitado, y sistema de gestión que incluyan herramientas, metodologías e infraestructura adecuada que permita asegurar procesos de calidad, eficaces y eficientes basados dentro un modelo de gestión por competencias, que permita reclutar, seleccionar y contratar al talento humano, que cuente además de las aptitudes y actitudes, con las habilidades y destrezas necesarias para estar acordes y alineadas con las competencias que exige el cargo o posición dentro de la organización.

"Hay que reinventar recursos humanos, reforzando las competencias para generar valor. Una de las más grandes necesidades en el nuevo mundo del trabajo es la de repensar como las organizaciones manejan, evalúan y recompensan a su gente" (Deloitte University Press, 2015, p.5); aspectos que implican considerar al capital humano como un recurso estratégico necesario al que debemos atraer, desarrollar y mantener dentro de la organización, considerando su valor estratégico para el cumplimiento de los objetivos y metas organizacionales y un factor de cambio y mejoras a nivel institucional.

A este respecto, los modelos de selección de personal por competencias facilitan el desarrollo de proceso de evaluación, por lo tanto: "las competencias como características subyacentes del individuo que está relacionado con un rendimiento efectivo y/o desempeño superior en un trabajo o en una situación" (Spencer y Spencer, 2003, p.122). En este sentido, disponer de procesos de selección apropiados, permitan captar y seleccionar al recurso humano idóneo y competente, ya sea interno a través de la identificación del personal con talento humano dentro de la organización o la incorporación de personal externo competente, que en ambos casos, permitan generar un valor diferencial y de crecimiento organizacional, que posibilita además de su propio desarrollo personal y profesional y el desarrollo y crecimiento de la organización en su conjunto.

Las competencias se basan en el desarrollo de conocimiento, actitudes, motivaciones, y habilidades que nacen a partir de la interacción propia y la que se establece entre las personas, que, en algunos casos, se le denomina inteligencia emocional.

La inteligencia emocional, es la capacidad humana de sentir, entender, controlar y modificar los estados emocionales uno mismo y en su interacción con los demás, aspectos claves dentro de un 
proceso de gestión y relacionamiento dentro de cualquier organización, considerando las diversas interacciones y emociones que se generan dentro y fuera de la organización. (Goleman, 1996)

Por tanto, administrar el talento humano se convierte cada día en un reto, en la que las organizaciones deben tener una actitud abierta basada en la confianza hacia sus colaboradores, haciendo que se sientan motivados y comprometidos consigo mismos y con la organización y que sus competencias individuales estén alineadas a las competencias organizacionales, aspectos que permiten sincronizar sus objetivos, esfuerzos y metas individuales con los de la organización, situación que facilita el cumplimiento de los objetivos empresariales, la optimización del rendimiento de los trabajadores, y una ventaja competitiva frente a otras empresas del sector.

El capital humano constituye el recurso más estratégico, y también el más complejo de gestionar. El capital humano, esto es, el conjunto de habilidades, conocimientos y competencias de las personas que trabajan en la empresa, es una fuente incuestionable de ventajas competitivas a largo plazo. (López Cabarcos y Grandio Dopico, 2005, p.59)

Bajo este concepto entendemos con más claridad el valor estratégico del recurso humano dentro de la organización, que finalmente resulta ser una ventaja competitiva y diferenciadora dentro de la organización, es allí donde el recurso humano radica su importancia como talento humano, con características muy específicas relacionadas con el conocimiento, competencias y motivación, aspectos claves que deben incluirse con bastante precisión, dentro de las características y perfil del puesto requerido por la organización.

Tras la crisis, los departamentos de recursos humanos se enfrentan al reto de movilizar el talento de las empresas para apoyar los planes de crecimiento internacional. Esta situación representa, además, una gran oportunidad para convertirse definitivamente en el socio estratégico de la empresa, en un contexto en el que el talento se ha convertido en el factor crítico del éxito y la sostenibilidad, es la tarea prioritaria de los departamentos de recursos humanos. (Pin Arboledas y García Lombardía, 2014, p.14)

Algunas otras definiciones de competencias:

"La competencia hace referencia a las características de personalidad, devenidas comportamientos, que contribuyen a un desempeño exitoso en un puesto de trabajo. Cada puesto de trabajo puede tener diferentes características en empresa y/o mercados distintos" (Alles, 2006, p. 45).

"La competencia, se refiere a la capacidad de una persona para desempeñarse exitosamente en un trabajo, en un ambiente laboral favorable. La competencia es, entonces, una cualidad personal e intransferible que está referida a la realización de un trabajo determinado" (Martinez E. \& Martinez A., 2009, p.6).

De acuerdo a estas definiciones, debemos entender que una persona es competente cuando pone en práctica las competencias específicas en el desarrollo de sus funciones, que se exterioriza mediante comportamientos propios, como la motivación, actitudes, habilidades, y competencias específicas como la capacidad para identificar situaciones problemáticas, liderazgo y trabajo en equipo, creatividad, autocontrol, iniciativa, orientación al cliente, comunicación efectiva, ética, valores, entre otros dentro de su modelo de gestión para actuar y resolver problemas dentro de la organización y que son percibidos y evaluados por la misma organización.

Es claro, que no todos los seres humanos somos iguales, a pesar de las similitudes que podríamos tener con otros colegas, pero cada uno de nosotros tiene sus propias características y personalidad que marcan su propia diferencia y que se mantiene dentro de cada persona y estos son los aspectos que se pretenden revelar dentro de un proceso de selección por competencias, porque finalmente estas características son las que determinan su comportamiento y forma de actuar en el desarrollo de sus actividades, relacionamiento con sus colegas de trabajo, rendimiento y cumplimiento de objetivos organizacionales. 
El talento humano y competencias siempre se van a relacionar, por cuanto cuando se dice que una persona tiene talento, se relaciona con un desempeño superior al estándar y este se va enriqueciendo a través de conocimientos, competencias y motivación. Este talento innato o desarrollado se nutre de las competencias que cada persona va desarrollando que se exteriorizan mediante los comportamientos propios, que constituyen la parte visible de la competencia y generación del talento humano.

Los comportamientos representan lo que vemos en las personas y lo no visible son las competencias, es decir la parte visible de las competencias son los comportamientos. Actualmente, las organizaciones dan prioridad a las competencias que cada individuo tiene para así ocupe un puesto de trabajo y ayude en el logro y enriquecimiento de los objetivos organizacionales. (Alles M., 2006, p. 38)

"La existencia de altos índices de desempleo no significa que por ello es fácil la tarea de búsqueda y selección de personal. Los perfiles requeridos son complicados, y es complicado encontrar personas que lo cubran" (Alles M., 2006, p. 90). Esta definición hace referencia a la calidad antes que la cantidad de personas disponibles, que implica personas individuales con características y talento propio, y que este a su vez armonice con los requerimientos organizacionales, y la disponibilidad y disposición de estas personas hacia la organización.

Las competencias forman parte de la descripción del puesto. Cuando una empresa prepara su descripción de puestos de forma tradicional, se pregunta solamente, las tareas, obligaciones y responsabilidades que se requieren para desempeñarse en el puesto. En cambio, cuando se presenta una descripción de puestos basadas en competencias se pregunta, además, "cuáles son las competencias necesarias para ser exitoso en el puesto. (López Gumucio, 2010, p.134)

"Selección de competencias no significa, seleccionar jóvenes con potencial, ni profesionalismos universitarios, ni personas con alto promedio, selección por competencias, significa selección de personas con talentos, que le permite tener un mejor desempeño superior en su posición" (Alles M., 2006, p. 92). Estos conceptos, definitivamente guardan relación con procesos de selección basados en competencias, determinados por posiciones y perfiles por competencias que describan las características específicas de cada posición y la relación persona puesto que debe existir para ser exitoso en el puesto.

Las competencias dentro de su concepción y aplicación, hacen diferencias entre las habilidades duras (hard skills) y habilidades blandas (soft skills). Las primeras están referidas a las aptitudes, capacidades y habilidades técnicas específicas a cada puesto relacionadas con los requerimientos y competencias técnicas, mientras que las soft skills, hacen referencia a las características internas, personalidad, habilidades, actitudes y motivaciones personales relacionadas, que resultan más difíciles de determinar, pero ese es el reto.

Por diversos motivos, las organizaciones invierten de forma muy tímida en el desarrollo de sus equipos, ya sea por la inexistencia de estrategias sistematizadas de evaluación de desempeño, o por el desconocimiento de la importancia de la formación de un capital intelectual como factor diferencial. Las competencias de la organización están constituidas ante todo por la integración de las competencias individuales, lo que lleva a marcar la importancia que tiene para las organizaciones administrar tanto las competencias actuales como las potenciales. (Correa Arango y Miranda Martinez, 2013, p.32)

Es claro, que las competencias individuales se identifican a través del análisis de los comportamientos individuales, es decir lo que cada individuo aporta a la misión que le ha sido asignada realizándola de lo mejor posible, pero estos comportamientos individuales dentro de la organización se convierten en comportamientos organizacionales y por tanto, la organización lo que debe buscar es alinear estos comportamientos individuales con los comportamientos organizacionales, de manera de 
asegurar el cumplimiento de los objetivos y metas de la organización.

Las competencias, en función de la estrategia de cada organización se clasifican en genéricas y específicas. Las primeras referidas a un conjunto o grupo de actividades y las segundas destinadas a funciones o tareas específicas. Dicho de otra manera, las primeras se refieren a aquellos atributos o rasgos distintivos que requiere un trabajador excepcional en un puesto determinado. Estas incluyen conocimientos, habilidades, o actitudes específicas necesarias para desempeñar una tarea concreta. Las segundas son aquellos comportamientos observables y habituales que posibilitan el éxito de una persona en su función directiva. (Escobar Valencia, 2004, p.9)

La gestión organizacional conlleva de por sí el desarrollo de una serie de funciones y actividades con procesos propios e interrelacionados que determinan características propias a cada posición o puesto dentro la estructura organizacional. En este sentido, cada puesto dentro de la organización tiene competencias que son transversales a toda la organización y competencias específicas relacionadas con la posición específica; por tanto, los procesos de selección de personal por competencias, toman estos aspectos como elementos determinantes dentro de sus procesos de selección de personal.

Es por esta razón que la determinación del puesto y perfil constituye el elemento clave, base sobre la cual se lleva a cabo la búsqueda y captación de este personal con estas características específicas; búsquedas tanto internas como externas, que permitan identificar a este personal para poder invitarlos a participar en el proceso. Paso siguiente, es disponer de un proceso de evaluación previa para la preselección preliminar de candidatos que cumplan con los requerimientos del puesto y sobre esa base iniciar los procesos de evaluación, entrevistas por competencias y otros que corresponda, dependiendo del tipo de posición del candidato dentro de la estructura de la organización.

Las herramientas utilizadas para la selección de competencias:
- La entrevista focalizada/estructurada: Relacionada con una entrevista estructurada, que valora directamente si los candidatos poseen las competencias requeridas en los puestos.

- Pruebas o Test: Valora los aspectos de las competencias cognitivas, como, por ejemplo, Pensamiento analítico, Test de Personalidad: evalúa un gran número de competencias.

- Simulaciones, ejercicios prácticos y Assessment Center: Aporta gran valor añadido al proceso de selección, detectando competencias mediante una serie de actividades evaluadas por un panel de observadores.

- Información Bibliográfica: Estudio a través del currículum vitae, de situaciones pasadas en las que hubo más posibilidades de desarrollar unas determinadas competencias.

- Entrevista Telefónica: Comprobación de criterios que no pueden evaluarse en la entrevista curricular. Es un filtro indispensable en proceso de selección de gran volumen y en Assessment Center. (Becerra Gálvez y Campos Ahumada, 2012, pp.32-33)

Debemos entender que cada posición dentro de la organización tiene sus propias características y por tanto, las evaluaciones o entrevistas que se desarrollen están en función de estas características específicas y evaluación de competencias según cada caso. Concluida esta etapa, el Comité Evaluador evalúa todo el proceso y toma una decisión basada en asegurar la selección del mejor candidato que reúna las competencias, talento, y potencial para desarrollarse en la organización y generar valor a la empresa.

El proceso no culmina allí, porque una vez incorporado dentro sus responsabilidades en la organización, es necesario brindarle al nuevo personal las facilidades para su integración plena con los valores y objetivos organizacionales, que le permita además generar su propio desarrollo e identificación con la organización, alinear sus competencias personales con las 
competencias organizacionales, que asuman los objetivos de la organización como suyos, agregando valor y ventajas competitivas.

Asimismo, considerar que en el desarrollo de la actividad institucional, la persona es principal factor estratégico y ventaja competitiva con que cuenta una organización; por lo que la creación e implantación de un sistema de selección de personal por competencias permite alcanzar mayores niveles de competitividad, eficiencia, eficacia, productividad y satisfacción de los trabajadores en el desarrollo de la actividad laboral.

Es importante destacar que ningún modelo o tendencia teórica es superior a otra, simplemente lo que hay que analizar es la que mejor se adapte al tipo de organización y proceso; por tanto, considerando los resultados que esto genera para la organización, debe considerarse como parte de las estrategias empresariales prioritarias transversales a toda la organización. A este punto hay que agregar los impactos que genera contar con estos talentos humanos dentro de la organización permitiendo generar nuevos aprendizajes, nuevas formas de hacer las cosas y modelos de gestión innovativos que motiven a todo el personal a gestionar su propio cambio que genere su propio desarrollo y desarrollo de toda la organización en su conjunto.

Por tal motivo resulta importante relevar el rol prioritario y estratégico para toda organización, disponer de adecuadas políticas, modelos, procesos o sistemas de selección y desarrollo de personal por competencias, para asegurar no solo la selección del personal idóneo, sino también asegurar el desarrollo y retención del talento humano, sobre la base de generar las condiciones óptimas para lograr su plena satisfacción e identificación con la organización permitiendo su pleno compromiso y en conjunto alcanzar altos niveles de desarrollo y competitividad.

\section{CONCLUSIONES}

- La evolución de las organizaciones y la complejidad del mundo competitivo, ha conllevado de manera disruptiva a cambios radicales en la forma de gestión de las organizaciones, que ha traído consigo cambios importantes en la manera en la cual se recluta, selecciona y contrata al personal clave de las organizaciones, donde el elemento humano se convierte en el factor diferenciador entre el éxito y fracaso de una empresa, basado en sus competencias y talento humano.

- Para una adecuada selección de personal las organizaciones requieren disponer de procesos y herramientas adecuadas y validadas, que les permitan captar, entrevistar, evaluar y seleccionar a personal idóneo competente y con talento humano que se adecue a los requerimientos del perfil del puesto y que sus competencias personales estén acordes con las competencias organizacionales.

- En este sentido la selección por competencias dentro de un modelo de gestión por competencias, resulta determinante para la organización, por cuanto a través de este modelo de gestión, reclutar, seleccionar y contratar al personal competente más idóneo a la organización e internamente a nivel de la organización detectar las capacidades y habilidades existentes, que permitan seleccionar a ese talento humano que asegure un desempeño superior, basado en sus aptitudes y actitudes, motivaciones, relacionamiento y el control de los mismos, que se manifiesten en comportamientos presentes y futuros dentro de la organización en el desempeño de sus funciones y responsabilidades y niveles de relacionamiento interno y externo, alineados a los objetivos organizacionales.

- La importancia que significa para la organización contar con personal idóneo competitivo y con talento humano como elemento diferenciador que agrega valor $y$ se convierte en el elemento diferenciador y generador de impactos y ventajas competitivas a la organización. Internamente los impactos dentro de la organización generan aprendizajes y motivación a todo el personal a gestionar su propio cambio dentro de un ambiente laboral adecuado que facilite el desarrollo personal y profesional de todos sus integrantes y externamente los impactos de organización 
innovadora y competitiva que marca la diferencia y le permite un mejor posicionamiento dentro de su sector.

- La preocupación de la empresa por disponer cada vez más de personal competente o talento humano dentro de la organización, va a conllevar además de generar mayor productividad y competitividad, una mejor satisfacción laboral, mayor retención y menor rotación de personal y permitir el desarrollo y línea de carrera personal basado en la motivación y esfuerzo personal que conlleva trabajar con un buen clima laboral. A este efecto, resulta importante la elaboración de un plan de seguimiento permanente para valorar el grado de adecuación persona-puesto.

- Los impactos que genera le modelo de gestión por competencias en la selección de personal idóneo y competente, en relación a las personas genera mayor satisfacción laboral, aumento de la creatividad, mejor aprovechamiento de los recursos, más motivación, espíritu de equipo e inspiración, entre otros; en relación a la organización, impactos relacionados con el incremento de la eficiencia, mayor precisión estratégica, reducción del riesgo y mejora de la capacidad de empresa, y en relación a la sociedad, mejora en la calidad y oportunidad del servicio, ahorro de costos por eficiencia debido a un mejor rendimiento y una mayor rentabilidad económica y social.

Finalmente, en los tiempos actuales, el modelo de selección por competencias cobra mayor relevancia, si consideramos que son cada vez más las empresas que exigen profesionales con nuevas competencias y habilidades, que sean capaces de afrontar con éxito los retos del futuro, adaptarse a cambios internos y en su entorno y se encuentren adecuadamente preparados para transformar a las organizaciones en organizaciones del futuro.

\section{REFERENCIAS BIBLIOGRÁFICAS}

Alles, M. A. (2006). Selección Por Competencias. Buenos Aires: Granica.

Becerra Gálvez, M., y Campos Ahumada, F. (2012). El Enfoque por Competencias y sus Aportes en la Gestión de Recursos Humanos [Título de Psicólogo, Universidad de Chile]. Repositorio Institucional.

Correa Arango, Y., y Miranda Martinez, V. (2013). Diseño de un Modelo de Gestión por Competencias en la Empresa Eisenhower Martínez S.A[ Tesis de Administrador, Universidad de Cartagena]. Repositorio Institucional.

Deloitte University Press. (2015). Liderando en el nuevo mundo del trabajo. Tendencias Globales en Capital Humano 2015, 1-114.

Escobar Valencia, M. (2004). Las Competencias Laborales. Estudios Gerenciales, 1-25.

Goleman, D. (1996). La Inteligencia emocional. Barcelona: Kairos.

López Cabarcos, A., y Grandio Dopico, A. (2005). Capital Humano como fuente de ventajas competitivas. España: Gesbiblo.

López Gumucio, J. R. (2010). La Selección de Personal Basada en Competencias y su Relación con la eficacia Organizacional. Perspectivas, 13(26), 129-152.

Martinez E., E., y Martinez A., F. (2009). Capacitación Por Competencias. Santiago de Chile: Derechos Reservados.

Pin Arboledas, J. R., y García Lombardía, P. (2014). La Batalla Por el Talento. Global Thinking, 1-44.

Ruiz Ramirez, I. P., Ruiz Ramirez, A. K., y Martinez Zea, P. (2017). Gestión del Talento Humano en la Selección del personal administrativo. Primera edición.

Spencer y Spencer. (2003). Modelo Genérico de Competencias. New York: Wiley. 